\title{
Caracterização fenotípica de suínos crioulos da região Centro-Sul do Paraná, Brasil
}

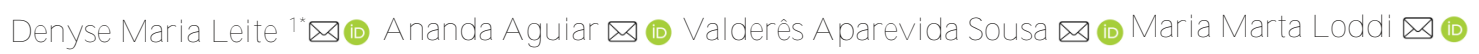

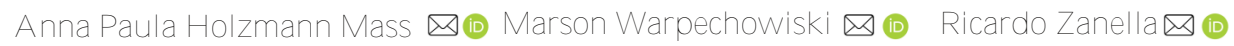 \\ Instituto de Desenvolvimento Rural do Paraná. Rua da Bandeira, 500, CEP:80035-270, Curitiba-PR, Brazil
}

\section{Phenotypic Characterization of Creole Pigs}

\begin{abstract}
A bstract: This research aimed to phenotypically characterize 55 pigs from traditional communities, called Faxinal with each pig, 24 variables were measured: 8 qualitative morphological, 16 quantitative/ zoometric and 5 zoometric indices were determined. According to the frequency tables of the qualitative variables, it was observed that most pigs showed similarities to the morphological patterns observed in the Brazilian breeds Moura, Piau and Canastra. For the data of the zoometric variables and the zoometric indices, descriptive statistical analysis and variance analysis were applied using two different sources of variation: sex and Faxinal. There was a great morphological variability of the studied creole pigs, with the highest values of the variation coefficients observed croup length and width, live weight and snout length. According to the averages obtained, the pigs were classified as elliptical, mesocephalic, longilinous, concavilinous. Significant differences between Faxinal were observed for croup width, body length, snout and croup, croup height and interorbital distance and cephalic, facial and pelvic indices. Significant differences between sexes were observed for withers height, snout length, shin girth and interorbital distance. There was significant between Faxinal *sex interaction for some zoometric variables, but not for the indexes. For body measurements, the correlations were high and significant, with the thoracic perimeter, abdominal perimeter and body length being the characteristics that best correlated with live weight. Cranial measurements (lengths of snout, ear and head and interorbital distance) were moderate to low. To infer the population structure, a statistical model was applied based on a bayesian approach according to the Structure Harvest software. It was possible to identify three phenotypic groupings, corroborating the results of qualitative variables. Genetic characterization studies should be carried out in these populations to serve as a basis for defining strategic actions for conservation and sustainable use of these genetic resources.
\end{abstract}

Keywords: conservation, genetic resources, morfology, zoometric measures.

Resumo: Esta pesquisa teve como objetivo caracterizar fenotipicamente 55 suínos provenientes de comunidades tradicionais, chamadas de Faxinal. Em cada suíno foram mensuradas 24 variáveis: 8 morfológicas qualitativas, 16 quantitativa / zoométrica e determinado 5 índices zoométricos. De acordo com as tabelas de frequência das variáveis qualitativas, observou-se que a maioria dos suínos apresentaram semelhanças aos padrões morfológicos observados nas raças brasileiras Moura, Piau e Canastra. Para os dados das variáveis zoométricas e os índices zoométricos foram aplicados a análise estatística descritiva e a de variância. Verificou-se grande variabilidade morfológica dos suínos crioulos estudados, sendo os maiores valores dos coeficientes de variação observados para comprimento e largura da garupa, peso vivo e comprimento de focinho. Através das médias obtidas os suínos foram classificados como elipométricos, mesocefálicos, longilíneos, concavilíneos. Foram observadas diferenças significativas entre Faxinal para largura de garupa, comprimento do corpo, focinho e garupa, al tura da garupa e distância interorbital, e dos índices cefálico, facial e pélvico. As diferenças significativas entre sexo foram observadas para altura da cernelha, comprimento do focinho, perímetro da canela e distância interorbital. Verificou-se interação significativa de Faxinal*sexo para al gumas variáveis zoométricas. Para as medidas corporais as correlações foram altas e significativas, sendo o perímetro torácico, o perímetro abdominal e o comprimento do corpo as que melhor se correlacionaram com o peso vivo. Já as medidas cranianas (comprimento do focinho, orelha e cabeça e a distância interorbital) foram de moderadas a baixas. Para inferir a estrutura populacional foi aplicado um modelo estatístico baseado em uma abordagem bayesiana de acordo com o software Structure Harvester. Foi possível identificar três agrupamentos fenotípicos, corroborando com os resultados das variáveis qualitativas. Estudos de caracterização genética deverão ser realizados nestas populações para que sirvam de base na definição de ações estratégicas para programas de conservação e uso sustentável desses recursos genéticos.

Palavras-chave: conservação, recursos genéticos, morfologia, medidas zoométricas.

Recibido: 2020-10-30. A ceptado: 2021-04-07

${ }^{1}$ A utor da correspondência: leited@gmail.com 


\section{Introducción}

As raças autóctones, também chamadas de crioulas, naturalizadas ou localmente adaptadas de suínos, existentes no Brasil, são procedentes das diversas raças europeias, asiáticas e algumas inglesas e americanas, trazidas pelos portugueses durante o período colonial. A partir da mestiçagem, do cruzamento, da consanguinidade e da seleção dessas raças, surgiram as raças brasileiras de suínos que foram uma importante fonte de alimentação para todo país até início do século XX. Entretanto, a partir das mudanças ocorridas na indústria, mercado e preferência do consumidor, os suínos crioulos foram desvalorizados e substituídos por raças exóticas mais produtivas, com menor quantidade de gordura na carcaça. Desde então, as raças brasileiras de suínos têm enfrentado uma drástica redução em número e diversidade e muitas estão sob ameaça de extinção (Macêdo et al., 2020). Segundo Mariante (2015), informações da base de dados global de recursos genéticos animal para a alimentação e agricultura da FAO mostra que $20 \%$ das raças que se tem informações são classificadas como ameaçadas de extinção, e que em nível mundial tem-se perdido uma raça ao mês. Portanto, há uma preocupação mundial com a redução e perda da biodiversidade associada ao aumento da seleção entre animais de produção (Zanella, 2020).

Nas diversas regiões do Brasil, os suínos crioulos têm um importante papel socioeconômico para os agricultores familiares de subsistência. Estes são, geralmente, criados em sistemas extensivos, sem nenhum controle zootécnico e é difícil encontrar nos rebanhos raças locais sem mistura de raças exóticas (Silva Filha et al., 2010; Leite, 2014; Burda et al., 2019; Macêdo et al., 2020). Na região Sul, especificamente no estado do Paraná, Leite (2014) verificou a presença de suínos crioulos em comunidades tradicionais, chamadas de Faxinal. N essas comunidades os animais são explorados sem acompanhamento técnico e manejados em sistema silvipastoril tradicional, onde parte da alimentação dos animais é oriunda dos recursos naturais, tais como, frutas silvestres, raízes, bulbos, pastagens natural e cultivada, insetos, minhocas, entre outros (Leite et al., 2009).

Apesar da importância sobre a conservação dos recursos genéticos ainda são poucas as informações, na literatura brasileira, sobre o efetivo do rebanho, localização geográfica, caraterização fenotípica e genética, e de desempenho produtivo dos suínos crioulos. Para McManus et al. (2010), um dos primeiros passos para caraterização destes recursos genéticos inclui o conhecimento das variações nas características morfológicas. Assim sendo, o objetivo do presente trabalho foi realizar a caracterização fenotípica das populações de suínos crioulos, provenientes de Faxinais, localizados na região Centro-Sul do estado do Paraná.

\section{M ateriales y M étodos}

A caracterização fenotípica foi realizada em suínos crioulos presentes nos "Faxinais Emboque" (FE) e "Marmeleiro de Baixo" (FMB), localizados na região Centro-Sul do estado do Paraná, nos municípios de São Mateus do Sul e Rebouças, respectivamente. $O$ município de São Mateus do Sul encontra-se a uma Latitude: $25^{\circ} 59^{\prime} 09^{\prime \prime}$ S e Longitude: $50^{\circ} 11^{\prime} 50^{\prime \prime}$ W, estando a uma altitude de $830 \mathrm{~m}$, e o de Rebouças a uma Latitude: $25^{\circ} 36^{\prime} 22^{\prime \prime}$ S e Longitude $50^{\circ} 41^{\prime} 37^{\prime \prime}$ W, a uma altitude de $815 \mathrm{~m}$. A temperatura média anual desses municípios situa-se em torno de $17^{\circ} \mathrm{C}$ a $18{ }^{\circ} \mathrm{C}$ e o índice pluviométrico anual varia de $1200 \mathrm{~mm}$ a 1 $800 \mathrm{~mm}$. Foram coletadas informações de uma amostra formada por 55 suínos, sendo 43 fêmeas (F) multíparas prenhas e lactantes e 12 machos (M) reprodutores. Deste total, 28 (19F e 9M) e 27 (24F e 3M) eram provenientes do FMB e do FE, respectivamente. Os dados foram obtidos por avaliação individual dos animais de acordo com os princípios éticos de experimentação da Comissão de Ética no Uso de Animais Domésticos - CEUA, protocolado sob o no 23/ 2014.
Em razão da inexistência de registros zootécnicos nos rebanhos, houve necessidade de definir alguns critérios, como a idade, preferencialmente acima dos 12 meses, informado pelos produtores, e de pelagem com predominância em cada Faxinal.

Foram coletadas informações de oito (08) variáveis morfológicas por animal, sendo elas: perfil cefálico, tipo de orelhas, cor da pelagem, mucosa e dos cascos; presença de cerdas e mamelas e número de tetas. Estas variáveis foram comparadas com o referencial morfológico das raças brasileiras de suínos descrita por EMBRAPA/ CENARGEN (1990).

Para a caracterização morfológica quantitativa foram mensuradas 16 variáveis zoométricas $(e m c m)$, conforme Revidatti (2009) Silva Filha et al. (2010), sendo elas: medidas cranianas - comprimento da cabeça (CC), focinho (CF), orelha (CO); largura da cabeça (LC), focinho (LF), orelha (LO) e distância interorbital (DIO) e medidas corporais - comprimento da garupa (CG), corpo (CCO), garupa (LG); altura da cernelha (AC) e garupa (AG); perímetro torácico (PT), 
abdominal (PA), canela (PC) e eso vivo (PV). As medidas foram realizadas com fita métrica $\mathrm{e}$ hipômetro e a metodologia das aferições estão detal hadas na tabela 1.

Tabela 1. Medidas zoométricas.

\begin{tabular}{ll}
\hline Variáveis Zoométricas & \multicolumn{1}{c}{ Descrição } \\
\hline & \multicolumn{1}{c}{ Medidas Cranianas } \\
\hline CC & Distância medida desde a protuberância occipital externa até a ponta do focinho \\
CF & Distância medida desde a sutura fronto-nasal até a extremidade do focinho \\
CO & Distância medida entre o ponto central da base da orel ha e o vértice da mesma \\
LC & Distância medida entre ambas as apófises do temporal. \\
LF & Distância medida entre a base de ambos os caninos. \\
LO & Distância medida entre ambas as bordas da orel ha. \\
DIO & Distância medida entre ambas as apófises do frontal. \\
\hline & \multicolumn{1}{c}{ Medidas Corporais } \\
\hline CG & Distância medida desde a tuberosidade ilíaca externa (ponta da anca) até a ponta \\
CCO & da nádega \\
LG & Distância desdea base do occipital atéa vértebra coccígea. \\
AC & Distância medida entre ambas as tuberosidades ilíacas externas. \\
AG & Distância medida desdeo o solo até o ponto mais culminante da cernelha \\
& Distância vertical existente desde o solo até o ponto de união da região lombar com \\
PT & a garupa \\
PA & Rodeando o corpo na região torácica \\
PC & Rodeando o corpo na região lombar \\
\hline
\end{tabular}

O peso dos animais foi estimado através da equação de regressão determinada para animais em idade reprodutiva ( $\geq 10$ meses), segundo metodologia de Mutua et al. (2011).

Peso Vivo Estimado $(\mathrm{PVkg})=0.35(\mathrm{CCO})+1.02(\mathrm{PT})-$ 74

A avaliação racial e funcional dos animais estudados foi desenvolvida a partir de seis índices zoométricos, conforme metodologia adaptada de Revidatti (2009) e Silva Filha et al. (2010). Os índices estão detalhados na tabela 2.

Tabela 2 - Índices zoométricos de suínos.

\begin{tabular}{ll}
\hline Indice Zoométrico & Descrição \\
\hline Índice Cefálico (IC) & LC / CC x 100 \\
Índice Facial (IF) & CF / CC x 100 \\
Índice Corporal (ICO) & COC / PT x 100 \\
Índice Pévico (IP) & LG / CG x 100 \\
Índice de Compacidade (ICD) & AC / PV x 100 \\
Índice de Carga da Canela (ICC) & PC / PV x 100 \\
\hline
\end{tabular}

Para os dados das variáveis qualitativas foram estabelecidas tabelas de frequência e das variáveis zoométricas e índices foram aplicados análise estatística descritiva e a de variância utilizando duas fontes de variação: sexo e faxinal, considerando um fatorial $2 \times 2$. Para todas as análises foi utilizado o Programa R. A significância das correlações fenotípicas foi verificada pelo teste $t$, baseado no intervalo de confiança de $99 \%$. Para inferir sobre os grupos de raças existentes no conjunto de indivíduos avaliados foi aplicado um modelo estatístico baseado em uma abordagem bayesiana de acordo com o software Structure 2.2.3 (Pritchard et al., 2000). A análise bayesiana foi realizada usando os modelos de fenótipos correlacionados com frequência de mistura com 50000 passos de Monte Carlo da Cadeia de Markov (MCCM) e 50000 períodos de "burn in" usando 500000 números de MCMC repetições. O número de $\mathrm{K}$ foi ajustado de 2 a 10 e 10 repetições foram executadas para cada $\mathrm{K}$. $\mathrm{O}$ número mais provável de clusters $(K)$ foi selecionado calculando a taxa de mudança de segunda ordem da função de verossimilhança em relação a K (Evanno et al., 2005) utilizando o software Structure Harvester (Earl, 2012).

\section{Resultados e Discussão}

A maioria dos suínos avaliados apresentaram perfis cefálicos retilíneo (49.09\%) e subconcavilíneo (49.09 $\%)$, orelhas ibéricas $(61.82 \%)$, com cerdas $(98.18 \%)$, cor de pelagem tordilha (47.27 \%) e oveira (32.73\%), cascos pretos (83.94\%), mucosa pigmentada escura, de cor preta (50.91 \%) e mesclada, de cor preta e branca (49.01\%), possuíam tetas variando de 12 a 14 (63.63\%) e sem mamelas (94.54 \%) (Tabela 3). Ao comparar os resultados das variáveis morfológicas estudadas com as descritas das raças brasileiras de suínos 
(EMBRAPA/ CENARGEN, 1990), constatou-se que a maioria dos suínos avaliados apresentaram semelhanças aos padrões observados, principalmente, nas raças Moura e Piau e poucos na raça Canastra (Figura 1). Diante deste resultado, é provável ter existido al gumas destas raças na formação dos grupos genéticos presentes nos Faxinais estudados. Em outros estudos, realizados no Brasil sobre caraterização fenotípica de suínos localmente adaptados, também foram observados a existência de suínos com algumas características morfológicas das raças brasileiras, tais como Moura, Piau, Canastra, Monteiro, Nilo,
Caruncho, Pirapitinga, Canastrão (Caval cante Neto et al., 2007; Silva Filha et al., 2010; Macêdo et al., 2020). Porém, nestas populações não foram verificados animais de raças puras e, provavelmente, no presente estudo. Esse fato pode ser devido aos cruzamentos desordenados de várias raças locais ou grupamentos genéticos e de raças comerciais introduzidas nos rebanhos. Por se tratar de cruzamentos sem acompanhamento técnico, a manutenção da diversidade genética pode ser comprometida, diminuindo as chances de sobrevivência dessas populações (Macêdo et al. 2020).

Tabela 3. Distribuição de frequência absoluta e relativa das variáveis qualitativas de suínos localmente adaptados, provenientes de Faxinais da região Centro-Sul do Paraná - Brasil.

\begin{tabular}{llrr}
\hline Variável Qualitativa & & Frequência A bsoluta & Frequência Relativa ( \%) \\
\hline \multirow{3}{*}{ Perfil Cefálico } & Retilíneo & 27 & 49.09 \\
& Subconcavilíneo & 27 & 49.09 \\
& Concavilíneo & 1 & 1.82 \\
\hline \multirow{3}{*}{ Tipo de Orelhas } & Ibérica & 34 & 61.82 \\
& Céltica & 17 & 30.92 \\
& Asiática & 4 & 7.27 \\
\hline Pelo & Presença & 54 & 98.18 \\
& Ausência & 1 & 1.82 \\
\hline \multirow{3}{*}{ Cor da Pelagem } & Oveira ${ }^{1}$ & 18 & 32.73 \\
& Tordilha & 26 & 47.27 \\
& Preta & 11 & 20.00 \\
\hline \multirow{2}{*}{ Cor dos cascos } & Preto & 46 & 83.64 \\
& Branco & 6 & 10.91 \\
& Preto ebranco & 3 & 5.45 \\
\hline \multirow{2}{*}{ Cor da mucosa } & Preta & 28 & 50.91 \\
Mamelas & Mesclada (preta e branca) & 27 & 49.09 \\
& Presença & 3 & 5.54 \\
& Ausência & 52 & 94.54 \\
\hline \multirow{2}{*}{ Número de tetas } & 08 & 4 & 7.27 \\
& 10 & 16 & 29.09 \\
& 12 & 26 & 47.27 \\
& 14 & 9 & 16.36 \\
\hline
\end{tabular}

1branca-bege com manchas pretas, toleradas manchas vermel has e brancas; 2preta entremeada de pel os brancos.
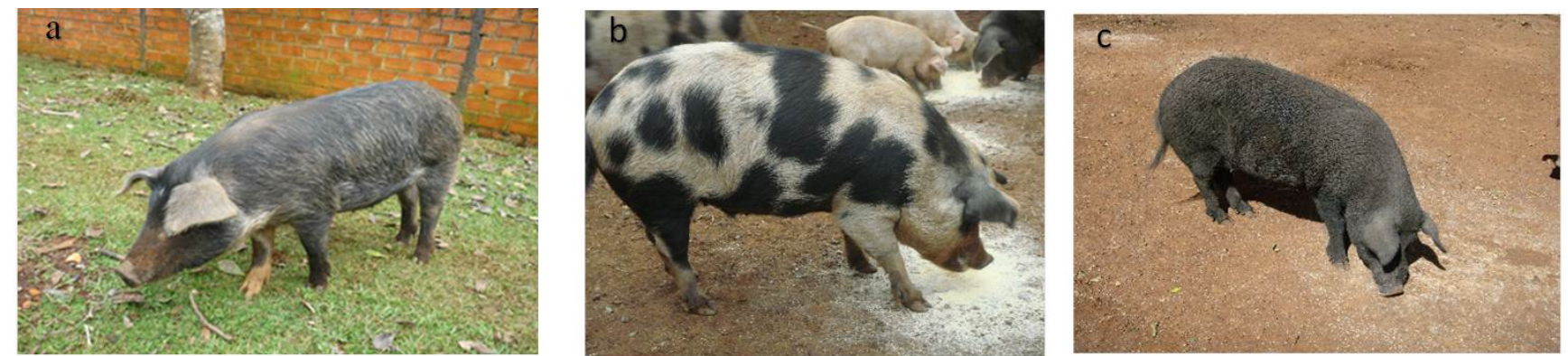

Figura 1. Exemplares de tipos de suínos que se assemelham as raças brasileira Moura (a), Piau (b) e Canastra (c), presentes nos Faxinais da região Centro-Sul do Paraná - Brasil.

De acordo com a tabela 4, verifica-se uma grande variabilidade morfológica dos suínos locais estudados, com os maiores valores dos coeficientes de variação observados para o comprimento da garupa (26.35\%), a largura da garupa (22.58 \%), o peso vivo (23.83) e o comprimento de focinho (20.54\%). Populações de suínos crioulos oriundos de outros países da América Latina apresentaram menores coeficientes de variação 
(Hurtado et al., 2004; Arredondo et al., 2011; Vargas et al., 2015; Céspedes et al., 2016, Velázquez et al., 2016) do que do presente trabalho. Esse fato pode ser atribuído aos sistemas de gestão e ambientes nos quais as populações foram desenvolvidas, diversidade genética existente entre elas, bem como o estado fisiológico dos animais. Os valores médios obtidos da altura da cernelha $(61.67 \mathrm{~cm})$ e do perímetro da canela $(17.16 \mathrm{~cm})$ foram próximos aos observados em populações de suínos crioulos do Brasil (Silvia Filha et al., 2010), assim como da Venezuela, Argentina e Equador (Hurtado et al., 2004; Revidatti et al., 2005). No entanto, em populações de suínos crioulos do Uruguaia, Castro et al. (2012) verificaram valores maiores. A altura da cernelha é uma característica pouco influenciada pelas condições ambientais em relação as outras medidas corporais, sendo assim importante para delimitar as diferenças ou semelhanças entre suínos provenientes de diferentes origens. O perímetro da canela também é uma das medidas importantes para o estudo morfológico de suínos, uma vez que o seu valor expressa o desenvolvimento esquelético com maior precisão do que qualquer outra medida. Em função do peso médio obtido (PV $=81.49 \mathrm{~kg}$ ), os suínos do presente estudo podem ser classificados como elipométricos ( $P V<180$ $\mathrm{kg}$ ) de tamanho pequeno. Resultado semel hante foram obtidos por Silva Filha et al. (2010) em populações de suínos crioulos no Nordeste do Brasil.

Tabela 4. Estatística descritiva das varáveis zoométricas e peso vivo da amostra total de suínos localmente adaptados ( $\mathrm{n}^{1}=55$ ), provenientes de Faxinais da região Centro-Sul do Paraná - Brasil.

\begin{tabular}{ccccc}
\hline $\begin{array}{c}\text { Variável zoométrica } \\
(\mathrm{cm})^{2}\end{array}$ & Média $\pm \mathrm{DP}^{3}$ & Máxima & Mínima & CV $\left.^{4} \%\right)$ \\
\hline AC & $61.67 \pm 6.58$ & 80.00 & 48.00 & 10.76 \\
AG & $64.51 \pm 9.10$ & 79.00 & 32.00 & 14.11 \\
CC & $31.12 \pm 5.30$ & 55.00 & 21.00 & 17.03 \\
CO & $18.78 \pm 2.77$ & 24.00 & 12.00 & 14.77 \\
CF & $16.65 \pm 3.41$ & 27.00 & 8.00 & 20.45 \\
CCO & $106.02 \pm 13.68$ & 148.00 & 76.00 & 12.90 \\
CG & $29.98 \pm 7.90$ & 52.00 & 19.00 & 26.35 \\
DIO & $11.89 \pm 2.38$ & 18.00 & 7.00 & 19.99 \\
LC & $18.64 \pm 3.17$ & 30.00 & 12.00 & 17.01 \\
LO & $14.85 \pm 2.33$ & 20.00 & 9.00 & 15.68 \\
LG & $33.94 \pm 7.66$ & 49.00 & 19.00 & 22.58 \\
LF & $9.29 \pm 1.76$ & 16.00 & 6.00 & 18.95 \\
PA & $127.34 \pm 16.95$ & 173.00 & 100.00 & 13.31 \\
PC & $17.16 \pm 1.99$ & 23.00 & 12.00 & 11.59 \\
PV $(\mathrm{kg})$ & $81.49 \pm 17.79$ & 122.56 & 54.76 & 21.83 \\
PT & $115.02 \pm 12.21$ & 150.00 & 94.00 & 10.61
\end{tabular}

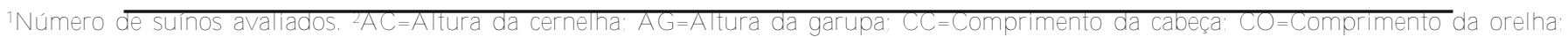
$\mathrm{CF}=$ Comprimento do focinho; $\mathrm{CCO}=$ Comprimento do corpo; $\mathrm{CG}=$ Comprimento da garupa; $\mathrm{DIO}=$ Distância Interorbital; $\mathrm{LC}=\mathrm{Largura}$ da cabeça; $\mathrm{LO}=$ Largura da orelha; $\mathrm{LG}=\mathrm{Largura}$ da garupa; $\mathrm{LF}=\mathrm{Largura}$ do focinho; PA=Perímetro abdominal; PC=Perímetro da canela; PT=Perímetro torácico; PV=Peso Vivo. ${ }^{3}$ Desvio-Padrão; ${ }^{4}$ Coeficiente de variação.

Em relação aos índices zoométricos (Tabela 5), verificou-se altar dispersão na maioria dos índices estudados, o que sugere que as populações avaliadas apresentaram um padrão racial heterogêneo. A partir dos valores médios obtidos dos índices zoométricos foi possível classificar os suínos da população estudada como mesocefálicos (IC $=64.69)$, longilíneos (ICO =93.98) e concavilíneos (IP = 119.73).

Em vários estudos latinoamericanos os suínos crioulos foram classificados como mesocefálicos, cujas médias de IC foram maiores que 50 (Revidatti et al., 2005; Montenegro et al., 2014; Pérez et al., 2015; Vargas et al., 2015). Já nas populações de suínos crioulos caracterizadas por Silva Filha et al. (2010), no Brasil, Hurtado et al. (2004), na Venezuela; Arredondo et al. (2011), na Colômbia; Jiménez et al. (2014), na Guatemala e Hernández-Baca et al. (2017), na Nicarágua, os animais apresentaram proporções dolicocéfalas (IC < 50). Os caracteres étnicos referidos pela cabeça, como o índice cefálico, têm sua importância etnológica, especialmente porque sua variação não é influenciada por fatores ambientais e de manejo (Herrera e Luque, 2009). 
Tabela 5. Estatística descritiva dos índices zoométricos da amostra total de suínos localmente adaptados ( $\left.\mathrm{n}^{1}=55\right)$, provenientes de Faxinais da região Centro-Sul do Paraná - Brasil.

\begin{tabular}{ccccc}
\hline Índice Zoométrico $^{2}$ & Média $\pm \mathrm{DP}^{3}$ & Máxima & Mínima & CV $^{4}(\%)$ \\
\hline IC & $64.69 \pm 13.43$ & 95.24 & 36.84 & 20.76 \\
IF & $53.93 \pm 9.77$ & 74.07 & 29.41 & 18.12 \\
IP & $119.73 \pm 39.43$ & 222.00 & 60.98 & 32.94 \\
ICO & $93.98 \pm 11.70$ & 121.31 & 72.09 & 12.45 \\
ICD & $75.68 \pm 10.35$ & 99.35 & 59.87 & 13.68 \\
ICC & $21.06 \pm 3.08$ & 29.22 & 14.69 & 14.62 \\
\hline
\end{tabular}

${ }^{1} \mathrm{~N}$ úmero de suínos avaliados. ${ }^{2} \mathrm{C}$ =́ndice cefálico; IF=́ndice facial; IPÁndice pélvico; ICO= Índice corporal; ICD=́ndice de compacidade; ICC=́ndice de carga de canela. ${ }^{3}$ Desvio-Padrão; 4Coeficiente de variação.

Em populações de suínos crioulos nicaraguenses e peruanos, Hernández-Baca et al. (2017) e Céspedes et al. (2016), respectivamente, observaram que os animais eram longilíneos. Estes resultados foram semelhantes ao verificado no presente estudo, onde os animais foram classificados como longilíneos. Por outro lado, em outros estudos da América Latina os suínos crioulos foram classificados como mesolíneos (ICO > 83 e < 89) e brevilíneos (ICO <83) (Hurtado et al., 2004; Revidatti et al., 2005; Lorenzo et al., 2012; Vargas et al., 2015; Pérez et al., 2015). Para o IP, o qual indica a relação entre a largura e comprimento da garupa, verificou-se que a largura predominou ao invés do comprimento da garupa (IP > 100), indicativo de que são animais de baixa capacidade reprodutiva, devido ao osso pélvico pequeno e reduzido, concordando com os resultados obtidos por Silva Filha et al. (2010) e Jiménez et al. (2014). De acordo Revidatti et al. (2005), o índice de compacidade (ICD) é um índice funcional de interesse na produção de carne, e o índice de carga de canela (ICC) mostra a harmonia entre a massa total do corpo (peso vivo) e a conformação das extremidades (perímetro da canela), ou seja, para maior peso, maior grau de robustez no animal examinado, manifestado concretamente pela força das suas extremidades. Os valores médios obtidos no presente estudo para ICD (75.68) e ICC (21.06) foram maiores do que os observados em suínos crioulos por Silva Filha et al. (2010). Diante desses resultados, pode-se inferir que os suínos do presente estudo possuem maior proporção esquelética, são mais robustos e, consequentemente, mais pesados do que os suínos do Nordeste do Brasil. Por outro lado, outros autores encontraram valores mai ores para ICC e ICD em suínos crioulos argentinos (Revidatti et al., 2005) e para ICD em suínos peruanos (Pérez et al., 2015).

$\mathrm{Na}$ tabela 6 verifica-se que no Faxinal Emboque (FE) os suínos apresentaram maior LG $(P<0.001)$, CCO ( $P<$ $0.01)$, DIO e CF $(P<0.05)$ do que aqueles oriundos do Faxinal Marmeleiro de Baixo (FMB). Por outro lado, verificou-se maiores médias para as variáveis AG e CG $(P<0.001)$ no FMB do que no FE. Silva Filha et al. (2010) também verificaram efeito significativo entre localidade para algumas variáveis zoométricas em suínos crioulos brasileiros, assim como, em outros países latinoamericanos (Pérez et al., 2015; Vargas; et al., 2015). Estas diferenças observadas entre localidades podem ser devido às adaptações desenvolvidas pelos animais, ao longo dos anos, em função dos ecossistemas de cada localidade. É importante mencionar que os recursos naturais presentes nas diferentes localidades / países são variáveis, o que pode ser vantajosamente explorado, resultando em um maior desenvolvimento corporal dos animais (Hurtado et al., 2004).

Foram observadas diferenças significativas entre sexos para as variáveis $P C(P<0.001)$, AC e CF $(P<$ $0.01)$ e DIO ( $P<0.05)$, sendo as maiores médias observadas nos machos (Tabela 6). Este fato poderia ser uma indicação de dimorfismo sexual, mas devido ao baixo número de animais amostrados, isso não pode ser afirmado, e é desejável aumentar o número de machos amostrados. No entanto, McManus et al. (2010) encontraram dimorfismo sexual em variáveis cranianas e corporais, quando avaliaram raças crioulas do Brasil, Uruguai e Colômbia.

O efeito significativo encontrado da interação Faxinal*sexo para as variáveis AC e PC ( $P<0.001)$, AG e CCO $(P<0.01)$ e LF e PA $(P<0.05)$ pode refletir a interdependência desses fatores entre si, pelo fato do pastoreio ter sido praticado como um manejo comum nos dois Faxinais estudados, indicando que as forragens, embora não sendo o principal alimento para as espécies monogástricas, eram a base da alimentação dos animais. Diante desse resultado, sugere-se que a composição mista da oferta de alimentos foi variável nas diferentes localidades (gramíneas, leguminosas, raízes, tubérculos, frutos e outros). Hurtado et al. (2004) também verificaram efeito significativo da interação local x sexo para al gumas variáveis zoométricas. 
Tabela 6 - Comparação de médias das variáveis zoométricas e peso vivo de suínos local mente adaptados, provenientes da região Centro-Sul do Paraná - Brasil, por Faxinal (F), sexo (S) e interação FxS.

\begin{tabular}{|c|c|c|c|c|c|}
\hline \multirow{2}{*}{$\begin{array}{l}\text { Variável } \\
\text { Zoométrica }{ }^{1}(\mathrm{~cm})\end{array}$} & \multicolumn{2}{|c|}{ Faxinal } & \multicolumn{2}{|c|}{ Sexo } & \multirow[b]{2}{*}{$\mathrm{F} * \mathrm{~S}$} \\
\hline & $\begin{array}{c}\mathrm{FMB}^{2} \\
\left(n^{4}=28\right) \\
\mu^{5} \pm \mathrm{DP}^{6}\end{array}$ & $\begin{array}{c}\mathrm{FE}^{3} \\
(\mathrm{n}=27) \\
\mu \pm \mathrm{DP}\end{array}$ & $\begin{array}{c}\text { Fêmea } \\
(n=43) \\
\mu \pm D P\end{array}$ & $\begin{array}{r}\text { Macho } \\
(n=12) \\
\mu \pm D P\end{array}$ & \\
\hline$A C$ & $75.05 \pm 8.5$ & $76.28 \pm 11.7$ & $79.53 \pm 14.4$ & $79.05 \pm 7.3^{* k}$ & 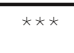 \\
\hline$A G$ & $68.2 \pm 6.5^{* 2 * k}$ & $60.7 \pm 9.8$ & $62.9 \pm 8.6$ & $70.3 \pm 8.1$ & ** \\
\hline $\mathrm{CC}$ & $31.6 \pm 4.6$ & $30.6 \pm 5.9$ & $30.7 \pm 5.5$ & $32.5 \pm 3.7$ & n.s. \\
\hline $\mathrm{CO}$ & $18.9 \pm 3.0$ & $18.7 \pm 2.5$ & $18.6 \pm 2.7$ & $19.4 \pm 2.7$ & n.s. \\
\hline $\mathrm{CF}$ & $15.7 \pm 3.8$ & $17.7 \pm 2.7 *$ & $16.9 \pm 3.0$ & $18.7 \pm 3.7^{* *}$ & n.s. \\
\hline $\mathrm{CCO}$ & $110.7 \pm 15.5$ & $101.7 \pm 13.5^{* *}$ & $104.9 \pm 12.2$ & $111.3 \pm 18.4$ & ** \\
\hline CG & $34.9 \pm 7.9^{\text {wak }}$ & $24.8 \pm 3.3$ & $28.9 \pm 7.6$ & $34.0 \pm 7.5$ & n.s. \\
\hline DIO & $10.7 \pm 2.0$ & $13.1 \pm 2.1^{*}$ & $11.7 \pm 2.3$ & $12.5 \pm 2.3^{*}$ & n.s. \\
\hline LC & $19.5 \pm 3.5$ & $14.7 \pm 2.6$ & $18.4 \pm 2.3$ & $19.4 \pm 1.9$ & n.s. \\
\hline LO & $15.0 \pm 2.4$ & $14.7 \pm 2.3$ & $14.7 \pm 2.3$ & $15.3 \pm 2.2$ & n.s. \\
\hline LG & $30.4 \pm 7.5$ & $37.6 \pm 2.3^{* 2 *}$ & $33.9 \pm 7.7$ & $34.0 \pm 7.0$ & n.s. \\
\hline LF & $9.5 \pm 1.9$ & $9.0 \pm 1.6$ & $9.1 \pm 1.7$ & $9.8 \pm 1.8$ & $*$ \\
\hline PA & $129.9 \pm 17.9$ & $124.7 \pm 15.8$ & $128.0 \pm 17.6$ & $124.9 \pm 13.4$ & $*$ \\
\hline PC & $17.04 \pm 1.60$ & $17.3 \pm 2.3$ & $16.8 \pm 1.58$ & $18.6 \pm 2.5^{* 14 k}$ & *⿻1k \\
\hline PT & $113.0 \pm 10.7$ & $117.0 \pm 13.5$ & $114.9 \pm 12.7$ & $115.4 \pm 9.99$ & $*$ \\
\hline $\mathrm{PV}(\mathrm{kg})$ & $81.83 \pm 14.3$ & $81.84 \pm 16.09$ & $81.00 \pm 15.29$ & $83.73 \pm 14.97$ & ** \\
\hline
\end{tabular}

${ }^{1} \mathrm{AC}=$ Altura da cernelha; $\mathrm{AG}=\mathrm{Altura}$ da garupa; $\mathrm{CC}=$ Comprimento da cabeça; $\mathrm{CO}=$ Comprimento da orelha; $\mathrm{CF}=\mathrm{Comprimento}$ do focinho; $\mathrm{CCO}=$ Comprimento do corpo; $\mathrm{CG}=$ Comprimento da garupa; $\mathrm{DIO}=$ Distância Interorbital; $\mathrm{LC}=$ Largura da cabeça; $\mathrm{LO}=\mathrm{Largura}$ da orelha; LG=Largura da garupa; LF=Largura do focinho; PA=Perímetro abdominal; PC=Perímetro da canela; PT=Perímetro torácico, PV=Peso vivo; 2Faxinal Marmeleiro de Baixo; ${ }^{3}$ Faxinal Emboque; ${ }^{4} \mathrm{~N}$ úmero de animais; ${ }^{5} \mathrm{Média}$; ${ }^{6}$ Desvio-padrão. Médias por faxinal e por sexo apresentam diferenças significativas: *** $(\mathrm{P}<0.001)$; **( $\mathrm{P}<0.01)$; *(P<0.05).

A través da tabela 7, verifica-se que houve diferenças significativas dos índices zoométricos entre Faxinal e sexo, mas não houve para a interação Faxinal*sexo. Os maiores valores médios dos índices cefálico, facial e pélvico $(P<0.001)$ foram observados no FE do que no FMB. Para o índice corporal (ICO), compacidade (ICD) e carga de canela (IDC) não houve diferenças significativas $(P>0.05)$.

Em outros estudos foram observadas diferenças significativas entre localidades para IC, IP e ICO (Hurtado et al., 2004), para IC, IP (Estupiñán et al.,
2009) e para IC, IP, ICD (Revidatti et al., 2005), em suínos crioulos da Venezuela, Equador e Argentina, respectivamente.

Com relação ao efeito do sexo (Tabela 7), verificouse diferença significativa apenas para IF $(P<0.001)$. Os machos e fêmeas foram classificados como mesocefálicos (IC > 50) e longilíneos (ICO > 89). No entanto, Céspedes et al. (2016) verificaram dimorfismo sexual para ICO em suínos crioulos do Peru, onde os machos foram classificados como longilíneos e as fêmeas mesolíneas.

Tabela 7. Comparação de médias dos índices zoométricos de suínos local mente adaptados, provenientes da região Centro-Sul do Paraná - Brasil, por Faxinal (F), sexo (S) e interação FxS.

\begin{tabular}{|c|c|c|c|c|c|}
\hline \multirow[t]{3}{*}{ Índice zoométrico ${ }^{1}$} & \multicolumn{2}{|c|}{ Faxinal } & \multicolumn{2}{|c|}{ Sexo } & \multirow{3}{*}{$\mathrm{F} * \mathrm{~S}$} \\
\hline & $\overline{F^{2} B^{2}\left(n^{4}=28\right)}$ & $\mathrm{FE}^{3}(\mathrm{n}=27)$ & Fêmea $(n=43)$ & Macho(n=12) & \\
\hline & $\mu^{5} \pm D^{6}$ & $\mu \pm \mathrm{DP}$ & $\mu \pm \mathrm{DP}$ & $\mu \pm D P$ & \\
\hline IC & $57.41 \pm 11.13$ & $72.2 \pm 11.4^{* \text { *k }}$ & $52.9 \pm 9.50$ & $57.5 \pm 9.40$ & n.s. \\
\hline IF & $49.6 \pm 9.7$ & $58.4 \pm 7.8^{* 4 *}$ & $66.2 \pm 13.60^{* * * *}$ & $59.3 \pm 10.60$ & n.s. \\
\hline IP & $87.4 \pm 12.1$ & $153.2 \pm 28.1^{\text {*k* }}$ & $124.0 \pm 40.00$ & $104.4 \pm 31.0$ & n.s. \\
\hline $\mathrm{ICO}$ & $87.87 \pm 8.24$ & $100.31 \pm 11.64$ & $93.36 \pm 11.80$ & $93.39 \pm 17.48$ & n.s. \\
\hline ICD & $70.1 \pm 18.5$ & $69.0 \pm 19.4$ & $70.0 \pm 19.70$ & $68.0 \pm 13.80$ & n.s. \\
\hline ICC & $19.1 \pm 4.9$ & $19.6 \pm 5.2$ & $19.4 \pm 5.20$ & $19.2 \pm 3.80$ & n.s. \\
\hline
\end{tabular}

1ICÁndice cefálico; IFf́ndice facial; IPÁndice pélvico; ICOÁndice corporal; ICD f́ndice de compacidade; ICC =́ndice de carga de canela; 2Faxinal Marmeleiro de Baixo; ${ }^{3}$ Faxinal Emboque; ${ }^{4} \mathrm{~N}$ úmero de animais; ${ }^{5} \mathrm{Média}$; ${ }^{6}$ Desvio-padrão. Médias por faxinal e por sexo apresentam diferenças significativas: *** $(\mathrm{P}<0.001)$; **( $<<0.01)$; *(P<0.05). 
Para as medidas corporais as correlações foram altas e significativas (99\%), sendo o perímetro torácico, o perímetro abdominal e o comprimento do corpo as que melhor se correlacionaram com o peso vivo (Figura 2). Já as medidas cranianas (comprimento do focinho, orelha e cabeça e a distância interorbital foram de moderadas a baixas. Essas diferenças são provavelmente devido ao fato de que as características da cabeça estão fortemente relacionadas à raça, enquanto as características corporais são fortemente influenciadas pelo ambiente, dependendo do regime alimentar e do sistema de criação (McManus et al., 2010).

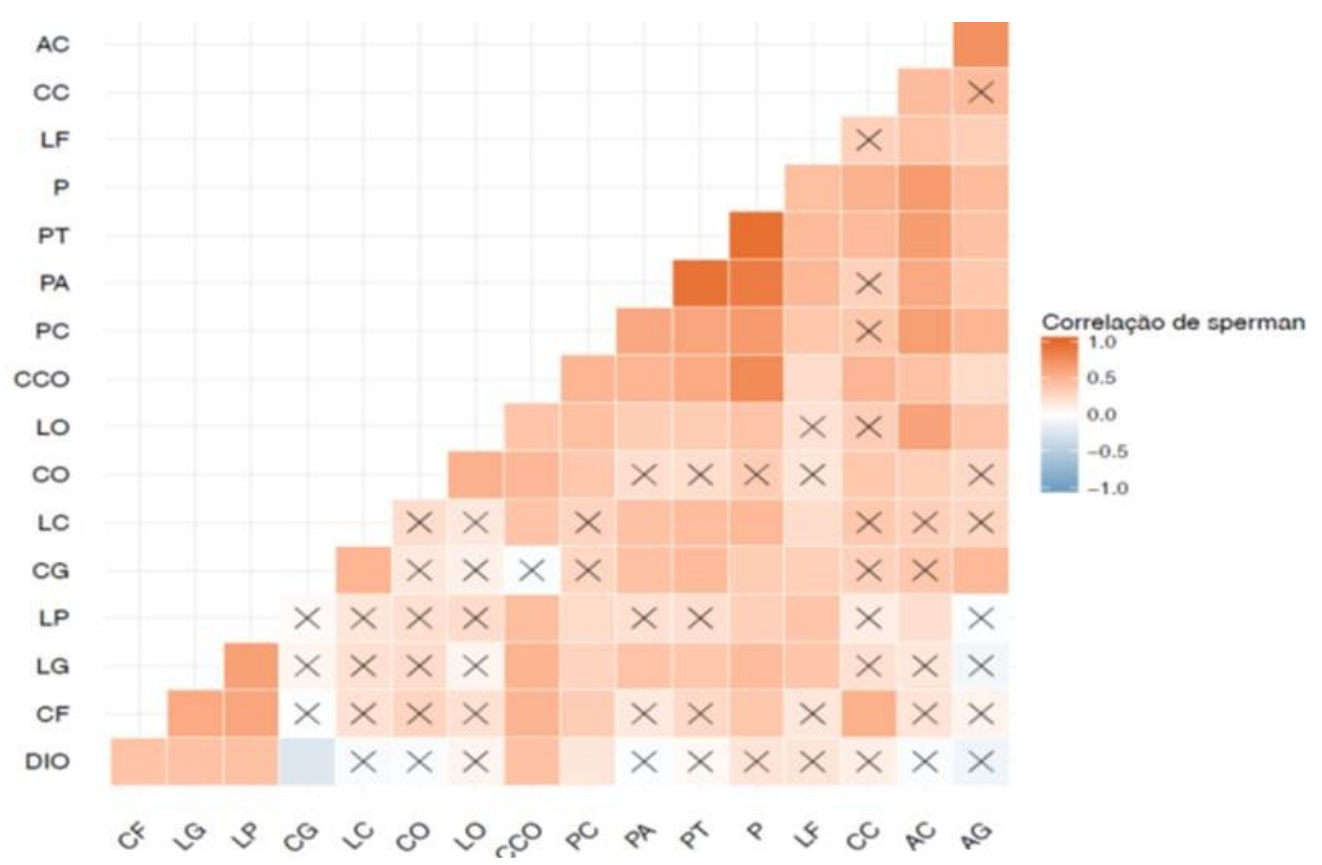

$\mathrm{X}$ - não significativa a $1 \%$ de probabilidade pelo teste $t . \mathrm{AC}=\mathrm{Altura}$ da cernelha; $\mathrm{AG}=\mathrm{Altura}$ da garupa; $\mathrm{CC}=\mathrm{Comprimento}$ da cabeça; $\mathrm{LF}=$

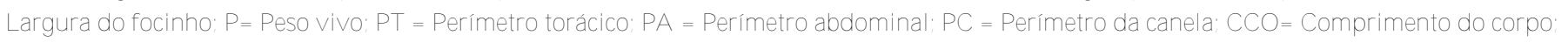
$\mathrm{LO}=\mathrm{Largura}$ da orelha; $\mathrm{CO}=$ Comprimento da orelha; $\mathrm{LC}=\mathrm{Largura}$ da cabeça; $\mathrm{CG}=$ Comprimento da garupa; $\mathrm{LP}=\mathrm{Largura}$ do pescoço; $\mathrm{LG}=$ Largura da garupa; $\mathrm{CF}=$ Comprimento do focinho; DIO =Distância Interorbital.

Figura 2. Correlação fenotípica das variáveis biométricas de suínos locais do Faxinal Marmeleiro de Baixo e Faxinal Emboque, localizados na região Centro-Sul do Paraná, Brasil.

As estimativas de diferenciação de grupo, a partir da abordagem bayesiana, confirmaram três agrupamentos fenotípicos de suínos nos Faxinais estudados (Figura 3). Esse resultado confirma os observados das variáveis morfológicas qualitativas. A nálise de estrutura onde $K=3$ teve a maior mudança na probabilidade posterior e em uma representação visual da estrutura a partir um gráfico populacional. Os valores de maior verossimilhança para as diferentes repetições (10) utilizadas na análise de atribuição bayesiana foram observados para $\mathrm{K}=3$ que contribuíram para um $\Delta \mathrm{K}$ alto (10.313958).
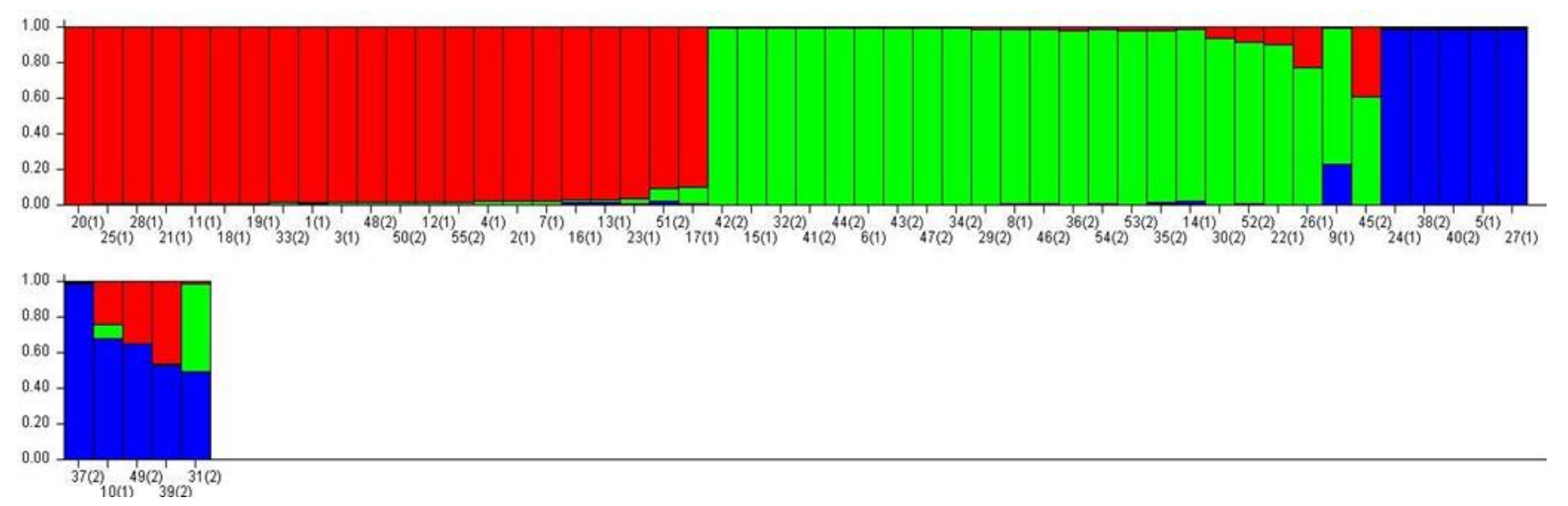

Os números fora dos parênteses refere-se à identificação do animal; (1) Faxinal Marmeleiro de Baixo; (2) Faxinal Emboque.

Figura 3 - Análise bayesiana das variáveis fenotípicas avaliadas em suínos locais do Faxinal Marmeleiro de Baixo e Faxinal Emboque, localizados na região Centro-Sul do Paraná. 


\section{Conclusões}

Os suínos avaliados diferenciaram quanto sua caracterização fenotípica, indicando possíveis agrupamentos populacionais independentes, que só poderão ser identificados após uma sequência de pesquisas de caracterização desses grupos, especialmente a genética. Esta análise é uma primeira abordagem da morfologia dos suínos crioulos presentes em Faxinais. Portanto, é necessário realizar estudos de caraterização genética destas populações para que sirvam de base na definição de ações estratégicas para programas de conservação e fomento.

\section{Literatura Citada}

Arredondo, J. V., J.E. Muñoz, L.E. Arenas, E. Pacheco, L.A. Álvarez. 2011. Caracterización zoométrica de cerdos criollos en el departamento del Chocó Colombia. Actas Iberoamericanas de Conservación Animal, v.1, p.57-59. https:/ / ai carevista.jimdo.com/ app/ download/ 129 82627625/ Arredondo2011_1_5_59.pdf?t=1610047402

Burda, J., M.B. Warpechowski, J.S. Brum, C.O. Novinski, M.M. Loddi, D. M. G. Leite. 2020. Caracterização do sistema de criação de porcos da raça Moura no Sul do Brasil. Anais do XX Simpósio Iberoamericano sobre Conservação e Uso de Recursos Zoogenéticos Locais. Corumbá, Brasil. p.44.

https:/ / ainfo.cnptia.embrapa.br/ digital/ bitstream/ item/ 212162/ 1/ -A nais-XX-Simposio-Rec-

Zoogeneticos-DOC-163-2020.pdf

Castro, G., M. Montenegro, N. Barlocco, A.Vadell, R. Gagliardi, S. Llambí. 2012. Caracterización Zoométrica en el Cerdo Pampa Rocha de Uruguay (descriptiva primaria). Actas Iberoamericanas de Conservación Animal. v. 2, p.83-86. http:/ / www.uco.es/ conbiand/ aica/ templatemo_11 0_lin_photo/ articulos/ 2012/ Trabajo037_AICA2012. pdf

Cavalcante Neto, A., L.P.G. Silva, M.N. Ribeiro, J.F. Lui, J.G. Barbosa, S.T.R. Castro, G.J.G. Souza. 2007. Censo e caracterização fenotípica de suínos de grupos genéticos naturalizados brasileiros existentes no Estado da Paraíba. Biotemas, v. 20, p. 123-126. https:/ / periodicos.ufsc.br/ index.php/ biotemas/ art icle/ view/ 20627/ 18802

Céspedes, R.D., L.M. Huamán, F. Ticona, C.L. Hurtado, J.W. Gómez, N.C. Gómez. 2016. Caracterización morfológica morfoestructural y faneróptica del porcino criollo (Sus scrofa) de A purímac-Perú. Actas Iberoamericanas en Conservación Animal, v. 7, p. 48-52. https:/ / aicarevista.jimdo.com/ $\mathrm{n}$ \%C3 \%BAmeros/ vol \%C3 \%BAmen-7-2016/

Earl, D.A., 2012. Structure harvester: a website and program for visualizing Structure output and implementing the Evanno method. Conservation Genetic Resources. v.4, p. 359-361. https:/ / www.researchgate.net/ publication/ 287104 091_Structure_Harvester_a_website_and_program_f
_visualizing_STRUCTURE_output_and_implementi ng_the_Evanno_method

EMBRAPA/CENARGEN. 1990. Suínos Nacionais. Brasília-DF: Empresa Brasileira de Pesquisa Agropecuária EMBRAPA, $23 \mathrm{p}$.

Estupiñán, K. V., D.V. Mora, S. Barreto, K. Zambrano. 2009. Estudio morfoestructural de una población de cerdos naturalizados en los cantones valencia y la Maná, Ecuador. Ciencia y Tecnología. v. 2, p. 15-20. https:/ / revistas.uteq.edu.ec/ index.php/ cyt/ article / view/ 83

Evanno, G., S. Regnaut, J. Goudet. 2005. Detecting the number of clusters of individuals using the software structure: a simulation study. Molecular Ecology. v.14, p. 2611-2620. https:/ / onlinelibrary.wiley.com/ doi/ abs/ 10.1111/ j .1365-294X.2005.02553.x

Jiménez, R.J., C.R.L. Machorro, L.V. Chegüen. Caracterización del cerdo Criollo de la región Chortí del departamento de Chiquimula, Guatemala. 2014. In: SILVA FILHA, O.L. Las razas porcinas Iberoamericanas: un enfoque etnozootécnico. Salvador, BA (Brasil): Instituto Federal de Educação, Ciência e Tecnologia Baiano (IF Baiano), p. 231-235.

Hurtado, E., C. González, J. Ly. 2004. Morfología del cerdo Criollo Venezolano del Estado Apure, Venezuela. Revista Computadorizada de Producción Porcina. V.11, p. 39-47.

Herrera, M., M. Luque. 2009. Morfoestructura y sistemas para el futuro en la valoración morfológica. Capítulo 14. p. 83-102. In: Sañudo, A. C. Valoración Morfológica de los animales domésticos. Ed. Madrid, Ministerio de Medio Ambiente y Medio Rural y Marino. p.863.

Hernández-Baca, M., A. Gámez-Rivas, Y. ZeledónOrtega. 2017. Caracterización morfológica del cerdo criollo (Sus scrofa domesticus) en el municipio de Nueva Guinea, RACCS. La Calera. v. 17, p. 21-27. https:/ / lacalera.una.edu.ni/ index.php/ CALERA/ a rticle/ view/ 281/ 287

Lorenzo, M., J. Jáuregui, C. Vásquez. 2012. Caracterización del cerdo criollo de la región cho'rti' del Departamento de Chiquimula, Guatemala. Actas Iberoamericanas de Conservación A nimal. v. 2, p. 103-108. 
http:/ / www.uco.es/ conbiand/ aica/ templatemo_1 10_lin_photo/ articulos/2012/ Trabajo048_AICA20 12.pdf

Leite, D.M.G. Caracterização do sistema de criação de suínos em Sistema Faxinal no Paraná, Brasil. 2014. In: Silva Filha, O.L. Las razas porcinas Iberoamericanas: un enfoque etnozootécnico. Salvador, BA (Brasil): Instituto Federal de Educação, Ciência e Tecnologia Baiano (IF Baiano), p. 337-368.

Macêdo, E.S., C.R. Silva, F.C. Lima, L.P. Costa, A. Macêdo, E.M. Brandão, E.F. Dias, A.C.G. Santos. 2020. Suíno baixadeiro: os suínos localmente adaptados da Baixada Maranhense - MA, Brasil. Revista de Recursos Genéticos - RG News, v. 6, p. 32-38.

http:/ / www.recursosgeneticos.org/ Recursos/ Arq uivos/6._Su_no_baixadeiro_os_su_nos_localmente _adaptados_da_baixada_maranhense.pdf

McManus, C., S. R. Paiva, A.V.R. Silva, L.S. Murata, H. Louvandini, G.P.B. Cubillos, G.O. Castro, R. A. Martinez, M. S. L. Dellacasa, J.E. Perez. 2010. Phenotypic characterization of naturalized swine breeds in Brazil, Uruguay and Colombia. Braz. Arch. Biol. Technol. v.53, p. 583-591. https:/ / doi.org/ 10.1590/ S1516-89132010000300011

Mariante, A.S. 2015. Plano de ação sobre recursos genéticos animais da FAO: um estimulo a conservação de raças localmente adaptadas. In: I Simpósio Internacional de Raças Nativas, Anais..., Teresina, PI.

Montenegro M., G. Castro, S. Llambí, N. Barlocco, A. Vadell, J.V. Delgado, C. Barba. 2014. Comparación de medidas zoométricas entre el cerdo Pampa Rocha y variedades oficialmente reconocidas del cerdo ibérico. Actas Iberoamericanas de Conservación Animal. v. 4, p. 86-88. http:/ / www.uco.es/ conbiand/ aica/ templatemo_1 10_lin_photo/ articulos/2014/ Trabajo119_AICA 201 4.pdf

Mutua, L.K., C.E. Dewey, S.M. Arimi, E. Schelling, W.O. Ogara. 2011.Prediction of live body weight using length and girth measurements for pigs in rural Western Kenya. J. of Swine Heal. Prod. v.9, p.26-33.

https:/ / www.researchgate.net/ publication/ 26562 6965_Prediction_of_live_body_weight_using_lengt h_and_girth_measurements_for_pigs_in_rural_We stern_Kenya

Pérez, F., A.C. Sierra, M.A. Canul, J.R. Ortiz, C.J. Bojórquez, J.C. Rodríguez, J. Tamayo-Canul. 2015. Caracterización etnológica del cerdo pelón en el Estado de Yucatán, México. Actas I beroamericanas de Conservacion Animal. v. 6, p. 443-451.
Pritchard, J.K., M. Stephens, P. Donnelly. 2000. Inference of population structure using multilocus genotype data. Genetics. v. 155, p. 945-959. https:/ / www.researchgate.net/ publication/ 106034 32_Inference_of_population_structure_using_multil ocus_genotype_data_Linked_loci_and_correlated_al lele_frequencies

Revidatti, M.A., J.V. Delgado, A. Capel lari, P.N. Prieto. 2005. Estudio morfoestructural preliminar de una población porcina en la provincia de Corrientes (Argentina). Archivos de Zootecnia, v. 54, p. 227232.

https:/ / www.researchgate.net/ publication/ 281046 94_Estudio_morfoestructural_preliminar_de_una_p oblacion_porcina_en_la_provincia_de_Corrientes_A rgentina

Revidatti, M.A.S. 2009. Caracterización de cerdos crioullos del Nordeste Argentino. Universidade de Córdoba (Espanha): Tese (Doutorado em Veterinária). 299p.

Silva Filha, O.L., E.C. Pimenta Filho, L.P.G. Silva, W.E. Pereira, R.J.F. Oliveira, J.V. Delgado, J.R.B. Sereno. 2010. Body morphometry of local pigs of Curimataú paraibano. Characterization of factors. Revista Computadorizada de Producción Porcina. v. 17, p. 197-202.

http:// www.iip.co.cu/ RCPP/ 203/ 203_02Gomesda Silva.pdf

Vargas, J.C., F.J. Velázquez, J.V. Delgado, Y. Sánchez. 2015. Caracterización zoométrica del cerdo criollo en los Cantones Mocache y Vinces, Provincia los Ríos, Región Costa, Ecuador. Actas Iberoamericanas de Conservación A nimal. v. 6, p. 562-570.

Velázquez, G.M., S.I.R. Ponce, A.V. Izquierdo, E.C. Torres, A.C. Covarrubias, L.C. Colíne, M.D. Aguilar, J.A.M. Jaquez, F.E.M. Silva, A.R. Utrera, V.E.V. Murillo, F.J. R. López. 2016. Morfometría del cerdo de traspatio en áreas rurales de México. Rev. Mex. Cienc. Pecu., v.7, p.431-440. https:/ / cienciaspecuarias.inifap.gob.mx/ index.php / Pecuarias/ article/ view/ 4276/ 3697

Zanella, R. 2020. The Use of Genomic Tools for A nimal Improvement and Conservation. Acta Scientific Veterinary Sciences, v.2, p. 01. https:/ / actascientific.com/ ASVS/ pdf/ ASVS-020096.pdf 